\title{
A study of the neurological disorder associated with acute haemorrhagic conjunctivitis due to enterovirus 70
}

\author{
NH WADIA, PN WADIA, SM KATRAK, VP MISRA \\ From the Departments of Neurology, Sir JJ Group of Hospitals, and the Jaslok Hospital and Research Centre, \\ Bombay, India
}

SUMMARY Ninety cases of the neurological manifestations associated with acute haemorrhagic conjunctivitis caused by Enterovirus 70 (EV 70) are reported. The patients were seen during the widespread epidemics in 1971 and 1981 . Male adults were predominantly affected by a "poliolike" paralysis of the limbs and/or cranial nerves. Root pains were often complained of early in the disease. In the absence of a necropsy, clinical and neurophysiological examinations helped to localise the lesions. Significant antibody titres against EV 70 were demonstrated in the serum and more relevantly in the CSF. Though other viruses can cause sporadic and epidemic conjunctivitis and similar paralysis independently, the combination of a haemorrhagic conjunctivitis and a neurological disease mostly simulating poliomyelitis is caused by EV 70 alone. It is therefore suggested that this combination be called "Enterovirus 70 disease". Because of its neurovirulence, it is important to identify this virus at the very beginning of an epidemic of conjunctivitis, so as to limit its spread by strict public health measures.

Amongst the new diseases known to have afflicted man in the last decade, acute haemorrhagic conjunctivitis stands out prominently. Its global incidence to date is hard to assess, but 70 to 80 millions in a decade would perhaps be an underestimate, considering reports of recent epidemics in the Indian subcontinent $^{12}$ and the Americas. ${ }^{3-5}$

During the 1971 pandemic, two independent observations were made which clearly indicated the aetiopathology of the disease. Wadia and his colleagues in Bombay ${ }^{67}$ showed that the disease was not confined to the eyes, but caused a "poliomyelitis-like illness" and Kono's group ${ }^{8}$ in Japan isolated a new enterovirus (EV 70) from the conjunctiva of Japanese patients. Neurovirulence of the virus in monkeys was shown, ${ }^{9}$ and the two groups jointly demonstrated that a single type of virus was responsible for the epidemics in Japan and India. ${ }^{10}$

As a large epidemic (May-October, 1981) of acute haemorrhagic conjunctivitis caused a similar illness, a comprehensive report of 90 cases of 1971

Address for reprint requests: Prof NH Wadia, Ben Nevis, B. Desai Rd, Bombay 400 036, India.

Received 31 May 1982 and in revised form 8 February 1983. Accepted 4 March 1983. and 1981 with new clinical and electromyographic data and some reference to virological observations is made here.

\section{Material and methods}

Twenty and 70 neurological cases respectively of 1971 and 1981 were clinically analysed. Whilst 84 suffered from acute haemorrhagic conjunctivitis, the other six (two in 1971 and four in 1981) were only in close contact with patients who had it, though their neurological illness was identical.

Detailed neurophysiological examination was carried out in 39 patients of 1981, and in five in whom it had been done in 1971. Using a 4-Channel DISA 1500 digital electromyograph, conventional electromyography (EMG) was performed of the clinically paralysed muscles and also of the adjacent non-affected and corresponding muscles of the opposite limb. Occasionally more distant muscles were also examined. Motor and sensory conduction was measured. Standard techniques were used. Amongst the special tests, $\mathrm{F}$ wave response was studied in $23, \mathrm{H}$ reflex in 23 , somatosensory evoked potentials (SEPs) in 18, and blink reflex in 20 . In 12 patients a second neurophysiological examination was performed between 9 to 19 weeks. F waves were recorded in the median, ulnar and peroneal nerves. Twenty supra maximal stimuli were given distally at intervals of 5 seconds. F wave latency, scatter and persistance were studied using the technique of Shahani et al. ${ }^{11}$ The percentage ratio of $F / M$ wave amplitude was nor 
studied consistently and is therefore not discussed. $\mathrm{H}$ reflex response was recorded from both lower limbs with the patient lying relaxed in the prone position and the feet just over the edge of the examination couch. With the pulse duration of the stimulus kept at $1 \mathrm{~ms}$, the common tibial nerve was stimulated at the knee with a gradually increasing current every 2 seconds. The shortest reproducable latency of the $\mathrm{H}$ response when recordable, and then with increased stimulus the shortest latency of the $M$ response, were measured. Blink reflex was recorded with surface electrodes. The cathode was placed over the lower lid just lateral to the middle of the eye, and the anode placed over the nasal bone. The supraorbital branch of the trigeminal nerve was stimulated in the supraorbital groove. One eye was studied at a time and an average of four epochs was taken as the final response. SEPs were recorded using subdermal DISA EEG needle electrodes over the leg and hand areas of the sensory cortex of the appropriate hemisphere. ${ }^{12}$ One hundred and twenty eight responses were averaged.

Eighty one samples of sera (single 31 , paired 22, triple 2) from 55 patients and 47 samples of CSF (single 31 and paired eight) from 39 patients, with appropriate controls were collected in the 1981 epidemic. These were sent to the National Institute of Health and Special Reference Laboratory, Tokyo, for estimation of antibody titres to EV 70 (J 670/71), three types of poliovirus (Mahoney, MEF , Saukett) and coxsackie A24 variant virus (EH 24).

\section{Results}

CLINICAL (Tables 1, 2, 3)

Age and Sex: Male adults predominated.

Prodromal symptoms: Well over half had fever and malaise, usually preceding, but at times continuing with the neurological symptoms. Those 46 who gave accurate information showed that the constitutional symptoms either accompanied the acute haemorrhagic conjunctivitis $(8.6 \%)$, or the neurological illness $(43.4 \%)$ alone, or were absent $(30.4 \%)$ or present $(17.6 \%)$ with both.

There were three main forms of the disease-the spinal, the cranial nerve and combined.

Spinal form: The illness often began with backache and muscular or root pains mostly in the legs. These were often severe and lasted at times up to three

Table 1 Distribution by age and sex

\begin{tabular}{|c|c|c|c|c|}
\hline \multirow{2}{*}{$\begin{array}{l}\text { Age groups } \\
\text { (In years) }\end{array}$} & \multicolumn{2}{|c|}{1971} & \multicolumn{2}{|c|}{1981} \\
\hline & $M$ & $F$ & $M$ & $F$ \\
\hline $\begin{array}{l}0-10 \\
11-20 \\
21-30 \\
31-40 \\
41-50 \\
51-60 \\
61-70 \\
\text { Total } \\
\text { Average age }\end{array}$ & $\begin{array}{l}1 \\
2 \\
7 \\
3 \\
3 \\
1 \\
20 \\
36 .\end{array}$ & $\begin{array}{l}- \\
\bar{Z} \\
2 \\
1 \\
-\end{array}$ & $\begin{array}{r}1 \\
1 \\
16 \\
20 \\
8 \\
7 \\
3 \\
70 \\
37\end{array}$ & $\begin{array}{l}-3 \\
3 \\
5 \\
2 \\
1 \\
\end{array}$ \\
\hline
\end{tabular}

Table 2 Clinical features (90 patients)

\begin{tabular}{|c|c|c|c|}
\hline & \multicolumn{2}{|c|}{ No of patients } & \multirow[t]{2}{*}{ Total } \\
\hline & 1971 & 1981 & \\
\hline $\begin{array}{l}\text { Acute haemorrhagic conjunctivitis } \\
\text { Prodromal Symptoms }\end{array}$ & 18 & 66 & 84 \\
\hline Constitutional & 7 & 43 & 50 \\
\hline Limb pains (muscular or root) & 13 & 41 & 54 \\
\hline \multicolumn{4}{|l|}{ Lower motor neuron paralysis } \\
\hline Lower limbs & 17 & 44 & 61 \\
\hline Diminished deep reflexes & 18 & 44 & 62 \\
\hline Facial & 1 & 28 & 29 \\
\hline Upper limb with lower limb & 1 & 23 & 24 \\
\hline Upper limb alone & 0 & 2 & 2 \\
\hline Trigeminal & 0 & 4 & 4 \\
\hline Abucens & 0 & 2 & 2 \\
\hline Vagus & 0 & 2 & 2 \\
\hline \multicolumn{4}{|l|}{ Mild, infrequent, transient features } \\
\hline Brisk reflexes; Extensor Plantars & 6 & 2 & 8 \\
\hline Cutaneous sensory loss & 0 & 8 & 8 \\
\hline Retention of Urine & 0 & 7 & 7 \\
\hline Vertigo & 0 & 4 & 4 \\
\hline Impaired vibration & 1 & 2 & 3 \\
\hline
\end{tabular}

Table 3 Forms of presentation. (90 patients)

\begin{tabular}{lrrr}
\hline & \multicolumn{2}{l}{ No. of patients } & Total \\
\cline { 2 - 3 } & 1971 & 1981 & \\
\hline Spinal & 19 & 36 & 55 \\
Facial & 1 & 20 & 21 \\
Spinal + facial & 0 & 6 & 6 \\
Spinal + trigeminal & 0 & 3 & 3 \\
Abducens & 0 & 2 & 2 \\
Trigeminal & 0 & 1 & 1 \\
Spinal + facial + vagus & 0 & 1 & 1 \\
Facial + vagus & 0 & 1 & 1 \\
\hline
\end{tabular}

weeks. The pains were accompanied or followed within 4 days by an acute essentially asymmetrical, proximal, hypotonic, areflexic paralysis of the lower limbs. The predominant symptom was a sudden buckling of the knees and inability to rise from the ground. The paralysis was often severe. The iliopsoas, the adductor longus and the quadriceps were most affected, followed by the lower paraspinal and abdominal muscles; the distal muscles were relatively spared. Upper limb weakness mostly followed the lower limb and was more frequent in 1981. Isolated upper limb paralysis was rare. Persistent wasting of the muscles was soon evident.

Cranial nerve form: Acute, isolated, unilateral lower motor neuron facial paralysis, often misdiagnosed as "Bell's palsy" was another notable form of the disease especially in 1981. Acute unilateral motor trigeminal, abducens and vagus paralysis was also seen.

Combined form: This was a variable combination of spinal and cranial nerve paralysis.

Infrequent signs: Table 2 also shows the infrequent and mild signs, which with rare exception, disappeared within days to weeks of the disease. Only one person had spastic paraplegia at onset along with 
lower motor neuron paralysis of face and right upper limb. The spasticity disappeared within two months, though the brisk reflexes and extensor plantars remained for some more time. The other seven had only extensor plantar responses and/or brisk deep reflexes. The cutaneous sensory loss was restricted to one or two lumbar roots. We never saw a transverse or ascending sensory loss. Short lasting vertigo only accompanied facial palsy. Urinary retention was seen only in those with severe lower limb paralysis.

Possible precipitating factors: Twenty of the 46 patients with limb paralysis had recently received intramuscular injections in the more paralysed limb. Four out of 14 women in 1981 were pregnant when they developed the paralysis.

Latent interval: The neurological symptoms followed acute haemorrhagic conjunctivitis in 83 of 90 patients. The latent interval (fig 1) in the spinal disease averaged 13.3 days in 1981 as against 3 weeks in 1971. It was only 6 days in those with isolated cranial nerve palsy. Interestingly, one of the remaining seven had acute haemorrhagic conjunctivitis 7 days after the spinal disease and six did not suffer from acute haemorrhagic conjunctivitis, but were in close contact with those who had it.

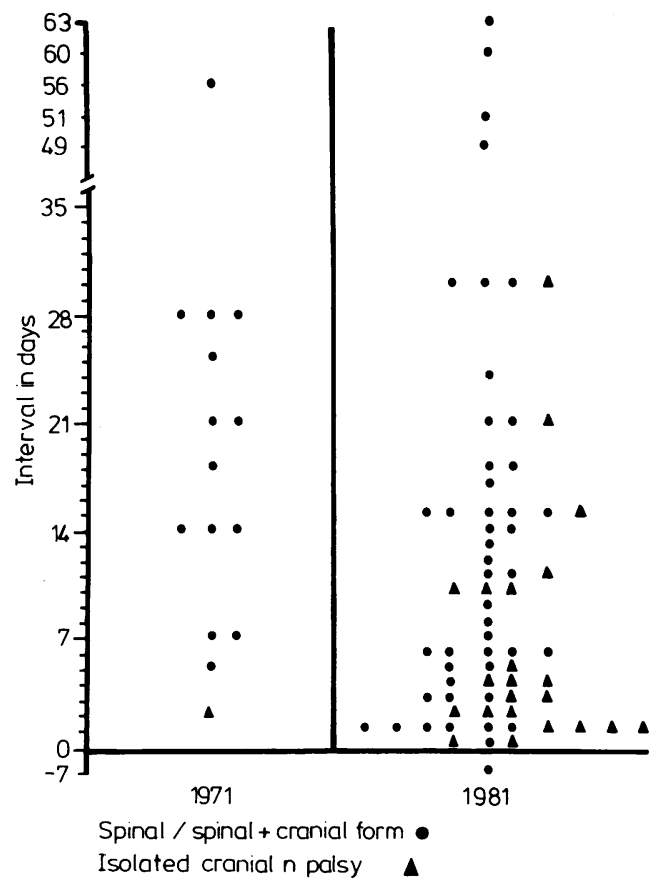

Fig. 1 Interval between acute haemorrhagic conjunctivitis and neurological disease. Zero represents onset of conjunctivitis.
CSF: The CSF was abnormal in 47 out of 61 during the acute stage of the spinal disease. It was examined in only seven with isolated cranial nerve palsy and was abnormal in three out of six when examined within 4 weeks of onset. The seventh was normal, but was examined on the 64 th day. In the majority, the cell count varied between 10 to 200 , mostly lymphocytes; pleocytosis has been absent in some. The cell count usually dropped to less than $10 / \mathrm{mm}^{3}$ by the fifth week. The initial protein level ranged from 40 to $400 \mathrm{mg} \%$ and remained high in some for several weeks giving a false impression of albuminocytological dissociation especially after the fifth week.

\section{NEUROPHYSIOLOGICAL DATA}

Electromyography: The first examination was carried out within 12 weeks of the onset of the paralysis. Spontaneous fibrillation and positive sharp waves began appearing as early as the first week in the affected muscles (figs $2 a, b$ ). On the whole, by the second or third week, a larger number of muscles showed spontaneous activity and in greater profusion. Widespread fasciculations were seen in only one patient. Clinically normal adjacent and/or corresponding muscles of the opposite limb also showed spontaneous activity (fig $2 c$ ). Occasionally such activity appeared even in the more distant unaffected muscles.

Where volitional effort was present normal motor unit potentials were first recorded. Long duration polyphasic potentials ( $>16 \mathrm{~ms}$ ) appeared as early as the second week (fig 3a). Late spikes were seen in some. Although an increase in the amplitude beyond $8 \mathrm{mV}$ (giant potentials) was seen in the second week in one patient, the number of large potentials increased significantly during the fifth week (fig 3a). Brief duration, small amplitude polyphasic

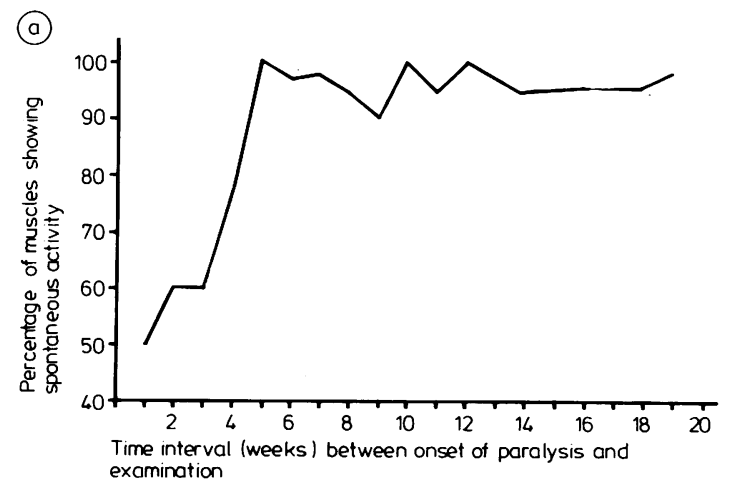

Fig. 2a Appearance of spontaneous activity in time during the first and/or second EMG examination. 


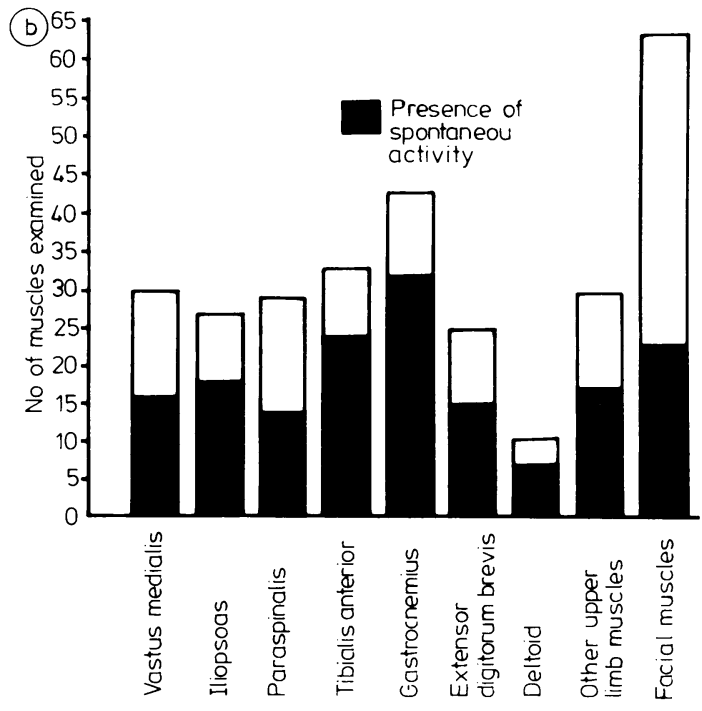

Fig. 2b Incidence of spontaneous activity in affected muscles within 12 weeks of paralysis.

potentials were seen in some of the muscles especially those of the face. Total or partial fall out of units was seen on maximal effort (fig $3 b$ ). Once again, in some adjacent and/or corresponding unaffected muscles of the opposite limb a reduced recruitment pattern was seen (fig $3 \mathrm{c}$ ).

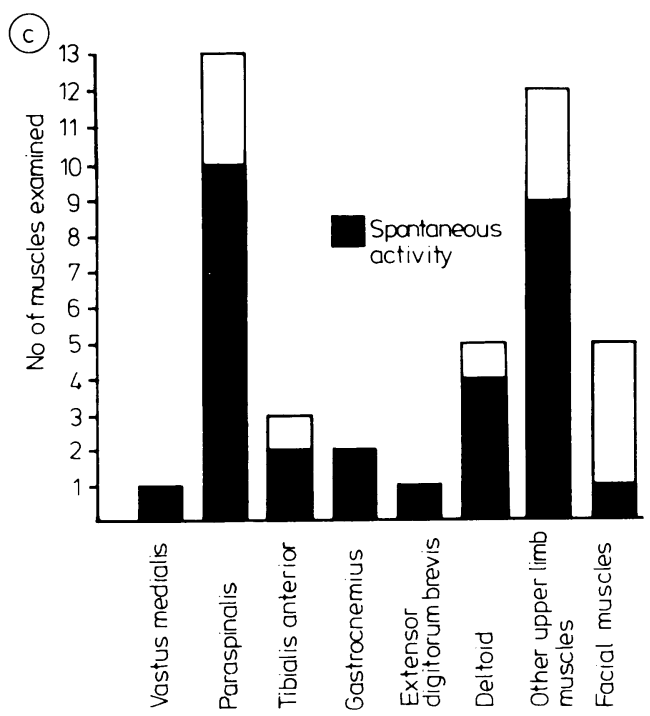

Fig. 2c Incidence of spontaneous activity in clinically unaffected adjacent or corresponding muscles of the opposite limb within 12 weeks of paralysis.

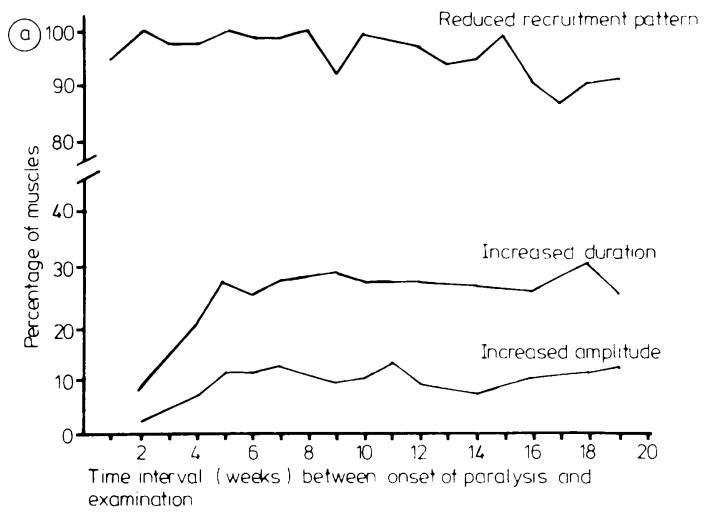

Fig. 3a Long duration large amplitude potentials on volitional effort mostly appear after the 5th week.

A second EMG was possible in 12 patients between 9 to 19 weeks. The incidence of spontaneous activity in most of the muscle groups earlier examined increased (fig 4) and there was a rise in the proportion of large polyphasic potentials (fig $3 a)$. The volitional activity remained the same or increased in some muscles (fig 5).

Nerve conduction velocity: Motor and sensory conduction velocity in the median, ulnar, peroneal and sural nerves was normal. No compound muscle action potential could be evoked following femoral nerve stimulation in five patients because of total

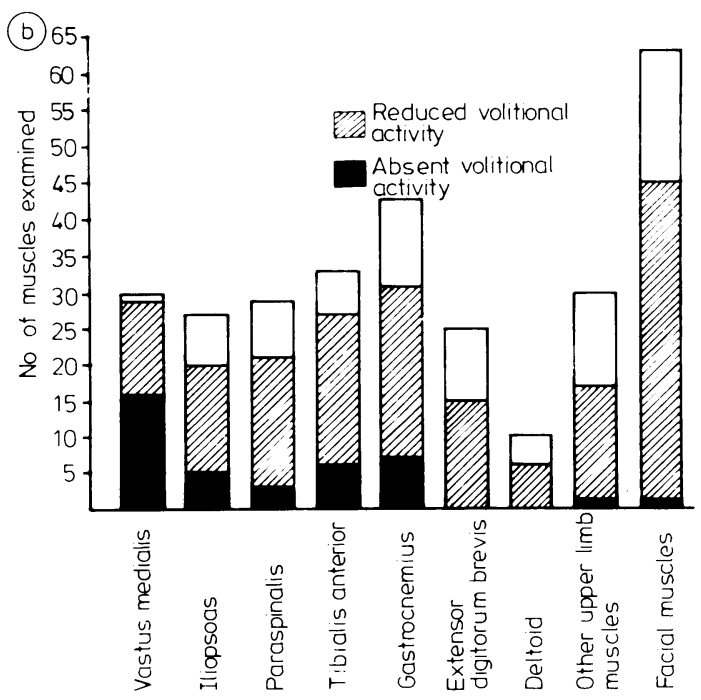

Fig. 3b Higher incidence of motor unit fall out (reduced volitional activity) in the vastus medialis within 12 weeks of paralysis. 


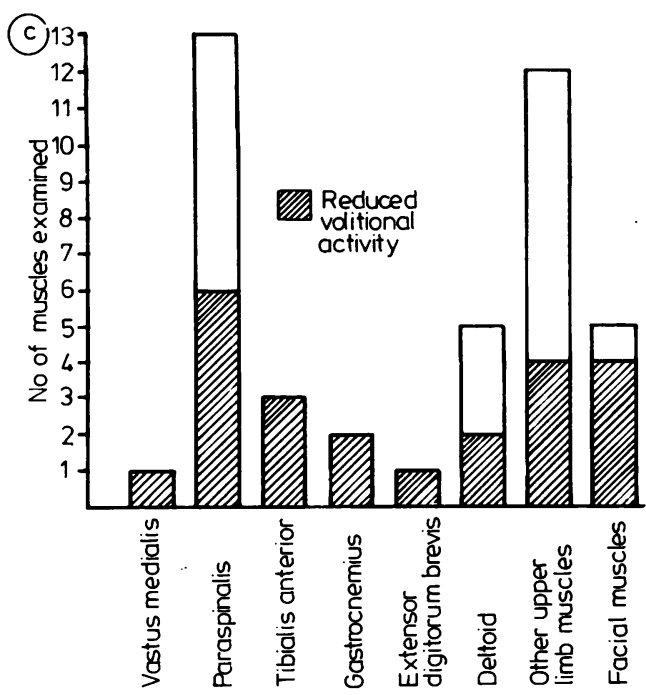

Fig. 3c Incidence of reduced volitional activity in the unaffected adjacent or corresponding muscles within 12 weeks of paralysis.

quadriceps paralysis. Conduction latency was slow in two and normal in another five (fig 6a).

Conduction latency in 16 of the 20 affected facial nerves was normal as compared to the unaffected side. Prolonged latency was seen in one or more branches to nine muscles of the other four nerves (figs $6 \mathrm{~b}, \mathrm{c}, \mathrm{d})$. Re-examination was possible in three

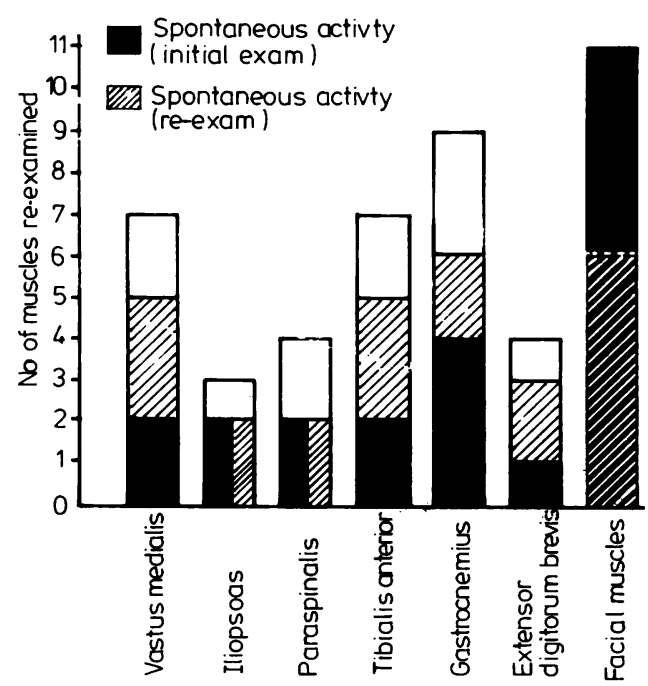

Fig. 4 Increase in the incidence of spontaneous activity on re-examination between the 9th to 19th week in all muscle groups except the facial.

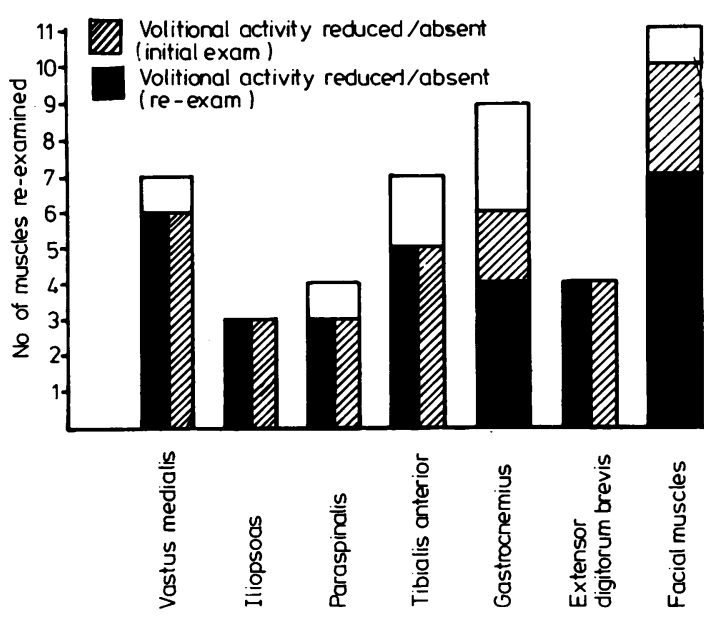

Fig. 5 On re-examination the volitional activity remained the same or increased as in the gastrocnemius and facial muscles.

patients and showed that the latency had returned to within normal range in five to 19 weeks, even as the face was clinically recovering.

$F$ wave response (figs $7 a b$ ): In the peroneal nerve, all parameters of the $F$ wave response were normal in seven of the 23 patients studied as standardised in our laboratory. It could not be elicited in six. Four showed abnormality of both the latency and scatter. In five, latency alone was abnormal and one had only an abnormal scatter. The conduction velocity was always normal. Three patients with abnormal $F$ wave response were re-examined at 9 to 19 weeks

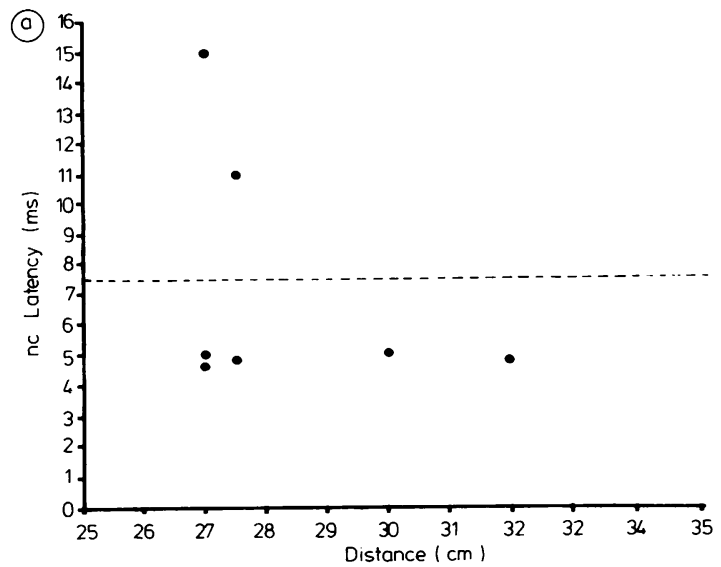

Fig. 6 Shows that the conduction latency in the femoral nerve was slow in only 2. The dotted line denotes the limit of normal. 

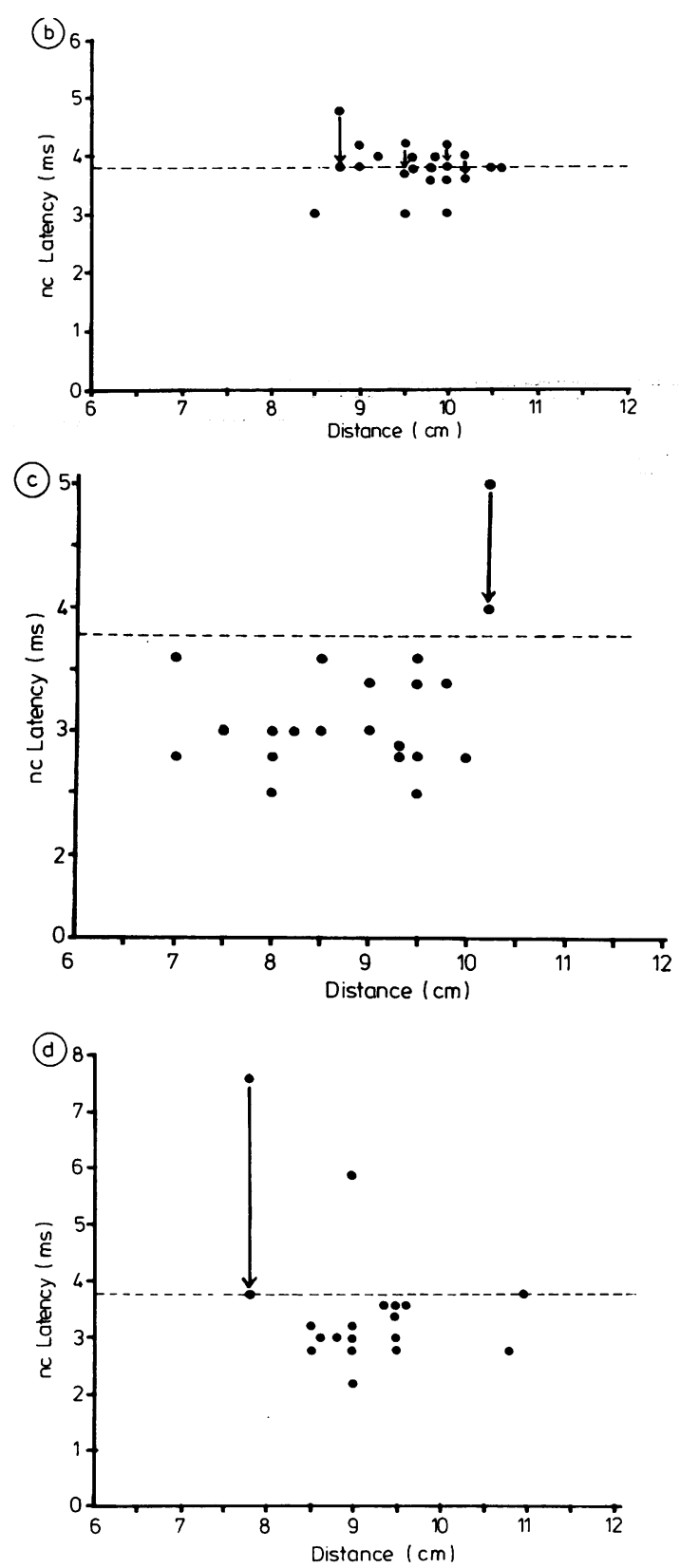

(b, c, d) show the conduction latency in the branches of the facial nerve to the frontalis, orbicularis oculi and orbicularis oris respectively. The dotted line denotes the limit of normal. Second examination (arrows) shows a shift towards normal.

after onset. In two where it was initially absent, a normal response, but with low persistence was recorded. In the other where the latency and scatter were abnormal, the $F$ wave became normal in all
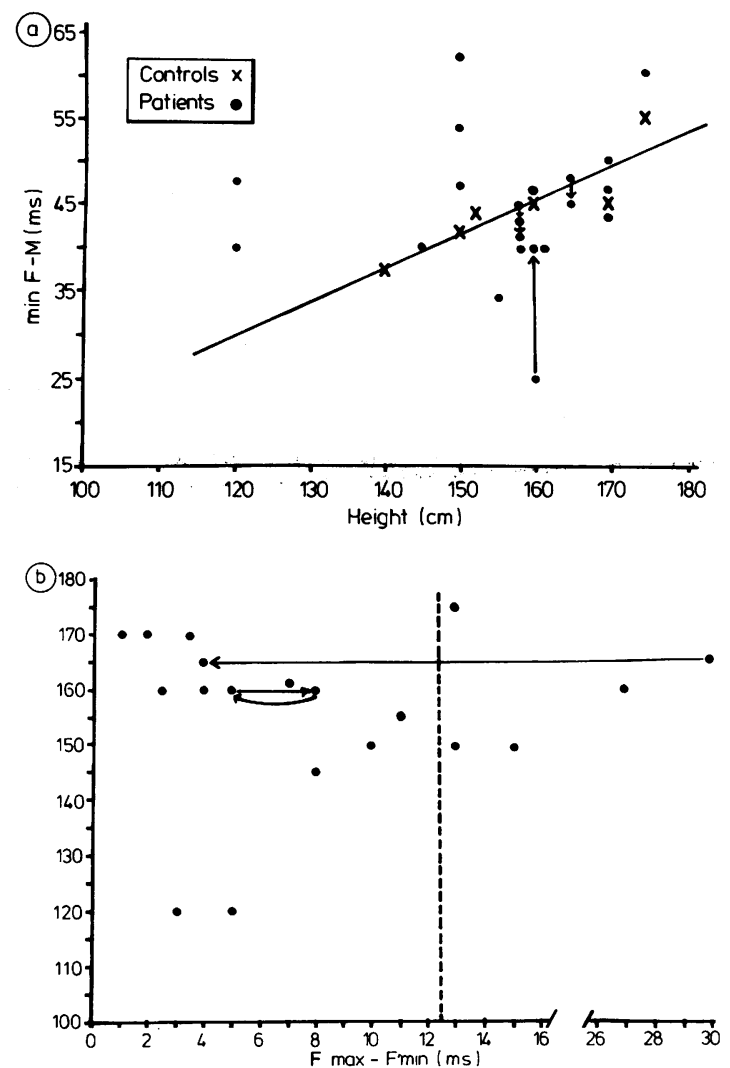

Fig. 7 (a) Shows the F latency (maximum $F$ latency - distal motor latency). The solid line represents normal values. Arrows indicate a second examination. (b) Shows the $F$ wave scatter (maximum $F$ latency-minimum $F$ latency). Arrows indicate re-examination. The dotted line denotes the limit of normal.

parameters. A normal $F$ wave response was recorded in the median and ulnar nerves of five patients with essentially proximal upper limb paralysis.

It is noteworthy that there was a close relationship between the $F$ wave response and paralysis of the muscle examined. The seven patients in whom the $F$ wave response was normal, had no weakness in the distal muscles, the proximal muscles alone having been clinically affected. Fifteen of the 16 patients whose EMG showed an abnormal $F$ wave response had clinical paralysis of the distal muscles of the lower limbs. The 16th patient had an abnormal $F$ wave response without clinical paralysis, but with evidence of denervation on EMG.

$H$ reflex: The $\mathrm{H}$ reflex was recorded in 23 patients with the spinal disease. It was normal in 14 and absent in nine. In four patients, in whom a re- 
examination was possible at 9 to 19 weeks after onset, the $H$ reflex remained absent. Whereas absent or present $\mathrm{H}$ reflex went hand in hand with an absent or normal ankle jerk respectively, in eight patients we were able to record a normal $\mathrm{H}$ reflex even when the ankle jerk was absent.

In eight of the patients with an absent $\mathrm{H}$ reflex, the $F$ wave was also abnormal. These patients were severely paralysed. In two of them the $F$ wave returned to normal in a later examination.

Somatosensory evoked potentials: SEPs was recorded from the lower limbs of 18 patients within the first 12 weeks. In nine patients they were recorded in both the lower limbs. They were abnormal in 12 as compared to our normal laboratory standards. In two SEPs were absent and in 10 were abnormal in form and latency (fig 8). The SEPs returned to normal in all three in whom a second examination was possible within 9 to 19 weeks of the onset. Eight of the 12 patients with abnormal SEPs had the initial sensory disorder like root pains and cutaneous proprioceptive loss, though it had disappeared by the time of the recording. On the other hand, only two of the six patients with normal SEPs had initial root pains, but no sensory loss. SEPs were recorded unilaterally or bilaterally from the median nerve of 10 patients and were normal in all, though three had root pains or sensory disturbance.

Blink reflex: The blink reflex was studied in 20 patients with facial palsy of varied severity and compared with our established laboratory standards and also with the opposite normal nerve. The patients could be broadly divided into three groups on the

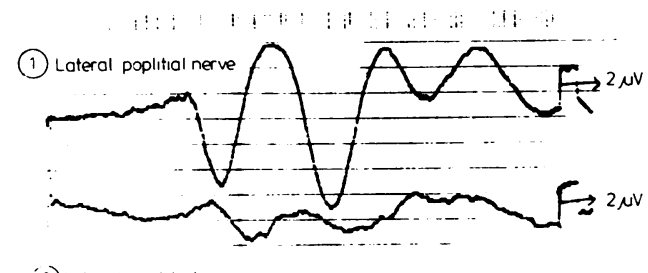

(2) Lateral poplitial nerve

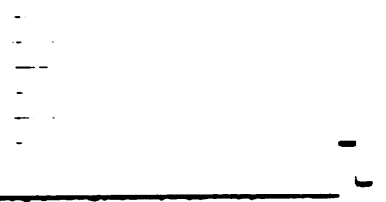

Fig. 8 Illustration SEPs. The upper tracing (1) is from the unaffected leg and the lower (2) from the affected. Each division on the time scale is equal to $1 \mathrm{~ms}$. Normal laboratory standards-lateral popliteal nerve. $N 1<$ $32.6 \mathrm{~ms}$. $\mathrm{P1}<39.6 \mathrm{~ms}$. N2 $<47.1 \mathrm{~ms}$. P2 $<56.4 \mathrm{~ms}$. Duration $<44.3 \mathrm{~ms}$. basis of the ipsilateral first response $\left(R_{1}\right)$ (table 4 ). In group I (three patients), the $R_{1}$ could not be recorded. In them, there was also prolongation of the inter-eye difference of the ipsilateral and consensual second responses $\left(R_{2}\right)$. In group II (eight patients) the $R_{1}$ was prolonged beyond $12 \mathrm{~ms}$ which was the upper limit of normal. In all of them the inter-eye difference of $R_{1}$ was always greater than $2 \mathrm{~ms}$. On the other hand, both $R_{2}$ responses were normal in latency in all patients. However, in two the inter-eye difference of the ipsilateral $R_{2}$ response was prolonged, whilst in one there was prolongation of the inter-eye difference of the ipsilateral and consensual $R_{2}$ responses. Group III (nine patients) had a normal $R_{1}$ response (under $12 \mathrm{~ms}$ ). However, it was more prolonged as compared with the unaffected opposite side, but the inter-eye difference was in the normal range. Except in one patient in whom the inter-eye difference of the ipsilateral and consensual $R_{2}$ responses was prolonged, all other parameters of the blink reflex were normal.

In all the patients with abnormal $R_{1}$ (Groups I and II) the conduction latency in the facial nerve was within normal limits except in one patient of Group II (fig 9).

One patient of group I was re-examined and the $R_{1}$ remained absent, but the prolonged inter-eye difference of the ipsilateral and consensual $R_{2}$ came back to normal. From group II, four patients were re-examined. $R_{1}$ was found to be still abnormal in one patient, but it had become normal in three (fig 9). The inter-eye difference of $R_{1}$ however continued to be abnormal in all four. The inter-eye difference of the ipsilateral and consensual $R_{2}$ returned to normal limits in all but one patient. $\mathrm{Re}$ examination of one patient in group III showed continued normality of the blink reflex. The nerve conduction latency was once again normal in all patients except one. However, it showed an improvement (fig 9).

We observed that the patients in groups I and II had severe facial paralysis and denervation with single oscillations. The paralysis was generally milder at the time of recording in group III. Only three had severe paralysis, and one of them had a prolonged inter-eye difference of the ipsilateral and consensual $R_{2}$ responses. The recovery was universally good in this group.

Virological data: So far no virus has been isolated from the biological material gathered from our patients. The significant rise of neutralising antibody titres in the sera of our 1971 patients against the Japanese strain of the acute haemorrhagic conjunctivitis virus (now called EV 70) has already been reported. ${ }^{10}$ Similarly diagnostic levels of antibody 
Table 4 Results of blink reflex study

\begin{tabular}{|c|c|c|c|c|c|c|}
\hline \multirow[t]{2}{*}{ Group } & \multirow{2}{*}{$\begin{array}{l}\text { No. of } \\
\text { patients }\end{array}$} & \multicolumn{2}{|l|}{$R_{1}$ response } & \multicolumn{3}{|c|}{ Inter-eye difference } \\
\hline & & $\begin{array}{l}\text { Affected side } \\
\text { (Latency, 8.2-12 ms)* }\end{array}$ & Unaffected side & $R_{1}(0-1 \mathrm{~ms})^{*}$ & $\begin{array}{l}\text { Ipsilateral } \\
R_{2}(0-3 \cdot 2 \mathrm{~ms})^{*}\end{array}$ & $\begin{array}{l}\text { Consensual } \\
R_{2}(0-3.5 \mathrm{~ms})^{*}\end{array}$ \\
\hline I & $\begin{array}{l}3 \\
5 \\
2 \\
1 \\
1 \\
8\end{array}$ & $\begin{array}{l}\text { Absent } \\
\text { Prolonged } \\
\text { Prolonged } \\
\text { Prolonged } \\
\text { Normal } \\
\text { Normal }\end{array}$ & $\begin{array}{l}\text { Normal } \\
\text { Normal } \\
\text { Normal } \\
\text { Normal } \\
\text { Normal } \\
\text { Normal }\end{array}$ & $\begin{array}{l}-\bar{P} \\
\text { Prolonged } \\
\text { Prolonged } \\
\text { Prolonged } \\
\text { Normal } \\
\text { Normal }\end{array}$ & $\begin{array}{l}\text { Prolonged } \\
\text { Normal } \\
\text { Prolonged } \\
\text { Prolonged } \\
\text { Prolonged } \\
\text { Normal }\end{array}$ & $\begin{array}{l}\text { Prolonged } \\
\text { Normal } \\
\text { Normal } \\
\text { Prolonged } \\
\text { Prolonged } \\
\text { Normal }\end{array}$ \\
\hline
\end{tabular}

* Our normal range.

Ipsilateral (21-40 ms) and Consensual (21-41.2 ms) $\mathbf{R}_{2}$ latencies were normal in all three groups in both eyes.

titres against EV 70 (prototype strain J 670/71) have also been demonstrated in the first batch of samples not only of sera, but of CSF of our 1981 patients. ${ }^{13}$ After this preliminary communication the rest of the material has been analysed and will be separately reported at length. ${ }^{14}$

In summary:

(a) All six samples of sera randomly collected from those not affected by acute haemorrhagic conjunctivitis during the epidemic did not show a significant level of neutralising antibody titres to EV 70 (J 670/71), (b) A significant level of antibody titres was seen (i) in seven of the 10 patients who had acute haemorrhagic conjunctivitis alone (ii) in 47 out of 51 who had acute haemorrhagic conjunctivitis and the neurological disease and (iii) in all four who had the neurological disease, but had not suffered from acute haemorrhagic conjunctivitis, though they

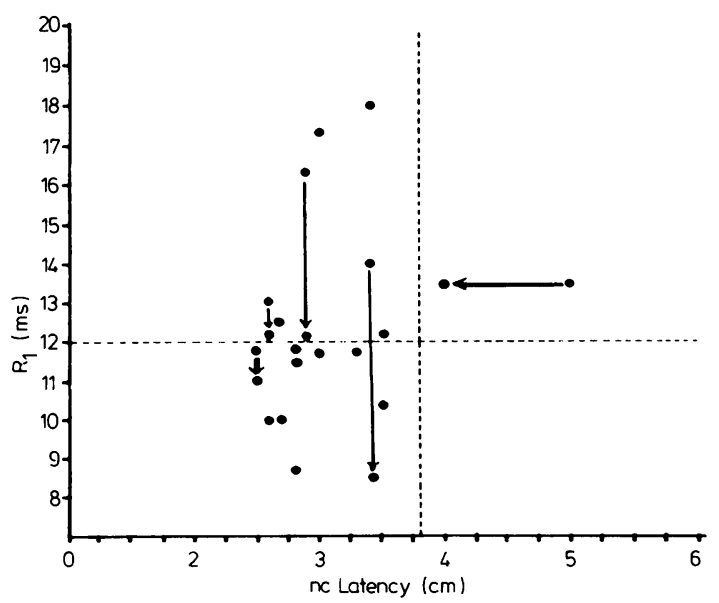

Fig. 9 Shows (i) Normal nerve conduction latency in facial nerve in all but one patient with prolonged $R_{1}$. (ii) On re-examination (vertical arrows) the $R_{1}$ in 3 patients returned to normal. (iii) Normal nerve conduction latency in all but one patient but with improvement on re-examination (horizontal arrow). Dotted lines show the limits of normal. were in close contact with those who had it. In paired or triple sera of 20 patients, rising or sustained high titres were found, (c) No significant antibody to EV 70 was seen in control samples of CSF from four patients who did not have acute haemorrhagic conjunctivitis and eight who had acute haemorrhagic conjunctivitis without the neurological disease. These control samples were necessarily drawn from patients whose CSF examination was done for some other disease such as stroke or brain tumour, (d) Similarly the CSF of 31 out of 39 neurological patients (both with the cranial nerve or spinal disease) showed a significant level of neutralising antibody titres $(>1: 2)$ and at times up to a maximum of 1:256. In paired CSF six showed rising or sustained titres, (e) Serum and CSF were simultaneously collected from 33 patients, and in 27 a significant rise of neutralising antibody titres was found in both fluids. The serum/CSF neutralising antibody titre ratio was significantly low and in fact the CSF titre was equal to or even higher than that in the corresponding serum in four patients, (f) No significant neutralising antibody titre was detected in the sera and CSF against the three types of polio virus and coxsackie A24 variant virus (EH 24).

Follow-up: A recent re-examination of nine of the 1971 patients with the spinal disease showed one to be permanently chair-bound, and six others had residual difficulty in arising. Two others had functionally recovered fully, though wasting and absent deep reflexes persisted.

A 3 month follow-up of 23 patients (13 spinal, five cranial nerve and five combined form of the disease) of 1981 showed recovery proportionate to the initial severity of paralysis. Though none had recovered fully, all had lesser grades of weakness. Recovery from cranial nerve paralysis was clearly more complete. At that stage, the disease had left five mildly, 15 moderately and three severely paralysed.

Electromyography performed on five of the 1971 patients showed persistent spontaneous fibrillation and long duration polyphasic potentials of normal to 
large amplitudes with fall out of units on maximum effort in the wasted and weak muscles. Giant potentials were recorded in muscles with good functional recovery, but there was little or no evidence of reinnervation where the recovery was poor. The motor and sensory conduction velocity remained normal.

\section{Discussion}

In 1971, when the first two patients were shown by one of us (NHW) to a group of Bombay neurologists, the condition was dismissed as fortuitous. Experience over the next 10 years showed that the recognition of these first cases formed the basis of the description of a new eye and nervous system disease caused by a new virus. By the end of the 1971 epidemic in India (a part of the pandemic) Wadia et al..$^{67}$ reported 19 patients with an acute polio-like illness following the conjunctivitis. They entitled it "lumbosacral radiculomyelitis", indicating that the main burden of the disease was on the anterior horn cells of the lower spinal cord, along with root involvement. No virus was isolated from these patients, but high antibody titres were found in their $\operatorname{sera}^{10}$ against the newly isolated acute haemorrhagic conjunctivitis virus (later called EV $70)^{8}$ from the conjunctiva of Japanese patients who had acute haemorrhagic conjunctivitis without any neurological illness. A case of facial palsy was also later reported. ${ }^{10}$ In the same epidemic, Bharucha and Mondkar ${ }^{15}$ saw somewhat similar cases in Bombay, though they stressed as much the sensory signs as the paralysis, and the rise in CSF protein without pleocytosis. They did not see the similarity to poliomyelitis and their cases were serologically unconfirmed. Bujarborua et al.$^{16}$ and Saxena et al. ${ }^{17}$ saw a few patients with acute cranial nerve palsy in North India. Cases were later reported from Taiwan ${ }^{1819}$ and Senegal ${ }^{20}$ by retrospective study of the 1971 pandemic, and from Thailand ${ }^{21}$ and Japan $^{22}$ in subsequent epidemics. Once again EV 70 was shown to be the cause of these various national epidemics ${ }^{19223}$ though no isolation of the virus from the CNS or demonstration of a significant antibody against EV 70 in the CSF was made.

The 1981 epidemic in India was on a vast scale, and some rough estimates place the total incidence of the eye disease at $\mathbf{4 0}$ million. It is likely that a large number of neurological cases have occurred, and effort is being made to gather more factual national data. Though conjunctivitis was prevalent at all ages in both sexes, male adults were predominant amongst those paralysed. No reason is known. Kono postulates higher immunity in children. ${ }^{24}$ Hung et al ${ }^{18}$ believe that male predominance is simply because there is greater prevalence of acute haemorrhagic conjunctivitis in males though other demographic surveys do not support this view. $^{325}$ We saw only one boy of 7 years in the two epidemics and enquiry amongst paediatricians did not reveal any increased incidence of "poliomyelitis" in children during 1981.

Whereas in the 1971 epidemic, the paralysis was observed to remain confined to the lower limbs, in the larger 1981 epidemic all combinations of limb and cranial nerve paralysis were seen. Yet the upper lumbar cord was most affected, and unlike cranial nerves, the upper limbs were rarely paralysed alone. We have never seen in such a short time so many cases of isolated facial nerve palsy, an experience shared by others. ${ }^{26-28}$ The close relationship to the conjunctivitis, the febrile malaise, the occasionally associated vertigo or the spinal disease and the CSF and electromyographic examination helped to rule out a coincidental Bell's palsy. An acute motor 5th nerve paralysis, a neurological rarity is recorded. We know of reported ${ }^{28}$ and unreported deaths from respiratory and bulbar paralysis, yet no clinically definite and virologically confirmed case has come to necropsy.

Clinically the main lesion is believed to be in the anterior horn cells of the spinal cord and the cranial motor neurons. Because the patients often had severe root pains at onset, the possibility of a concurrent radiculitis is also thought likely. The presence of evanescent pyramidal and sensory signs and vertigo further suggest reversible involvement of the nervous system besides the lower motor neuron. Serial neurophysiological examination was very helpful in diagnosis and localisation of lesions. The early appearance of widespread spontaneous activity followed by large polyphasic potentials and the recording of giant potentials after ten years of the disease, with normal conduction velocity indicate acute anterior horn cell disease with some recovery of the surviving neurons. The prolonged distal motor latencies in the femoral and facial nerves of a few of our patients is not incompatible with this conclusion, because similar observations have been made in poliomyelitis ${ }^{29}$ and amyotrophic lateral sclerosis $^{30}$ at a stage when the muscles are severely paralysed, as in our patients.

F wave abnormality with normal nerve conduction velocity has been reported as an early finding in demyelinating peripheral neuropathies like Guillain-Barré syndrome and diabetes mellitus, ${ }^{31}$ motor neuron disease ${ }^{32}$ and spinal muscular atrophy. ${ }^{33}$ However, in the majority of patients with peripheral neuropathy, the nerve conduction velocity soon becomes slower. Therefore, the abnormal F wave response in our patients along with persistently normal motor conduction would also indicate an 
acute anterior horn cell and/or anterior root disease. The normal $F$ wave response in the upper limbs probably suggests lesser involvement at least of the distal muscles.

The two consistent abnormalities in the blink reflex examination of the paralysed face were an absent or prolonged $R_{1}$ and an increased inter-eye difference of both $R_{1}$ and $R_{2}$ responses. With normal conduction latency these findings suggest a central nuclear lesion. In fact it proved useful in distinguishing these cases from coincidental Bell's palsy.

As would be expected serial examination of the blink reflex and $F$ wave response showed reversal of the abnormality as the paralysis lessened. Increased inter-eye difference seemed to be a more sensitive parameter in diagnosis than the abnormal $R_{1}$ response.

The abnormal SEPs and $\mathrm{H}$ reflex coupled with the normal sensory action potential suggest a lesion proximal to the dorsal root ganglion probably in the root entry zone, especially as the posterior columns and Clarke's column of cells do not form a part of the nervous pathway of the $H$ reflex and SEPs respectively. Thus abnormality of the $\mathrm{H}$ reflex study in an individual patient seems to result from a combination of a lesion in the root entry zone and the non-availability of a reflexly excitable motor neuron pool.

The fact that only eight of the 12 patients with abnormal SEPs had the initial sensory symptoms including root pain, and none had them at the time of recording points to the sensitivity of the test in documenting subclinical lesions. It is interesting that whereas in the majority the absent ankle jerk went hand in hand with an abnormal $\mathrm{H}$ reflex, in a few the latter was preserved when the former was absent. There is no ready explanation for that in this disease.

The overall clinical and neurophysiological examinations corroborate and suggest that the main impact of the disease is on the motor neurons of the lumbar and cervical cord and the cranial nerves. As in poliomyelitis ${ }^{34}$ evanscent root pains, occasional sensory disturbance, vertigo and pyramidal signs are also seen in the disease and probably result either from direct viral invasion of neurons or reversible tissue swelling adjacent to the main lesion. Besides this, the virus may have a longer reach as $w^{12}$ and others $^{283536}$ have seen a few cases of encephalitis, optic neuritis, papilloedema and acute polyneuritis simulating Guillan-Barré syndrome contiguous with the acute haemorrhagic conjunctivitis for which no alternate cause was found. Yet the small numbers do not rule out coincidental occurrence, specially in the absence of clear proof of virus invasion in these particular patients.
Although EV 70 was shown to be the causative agent of most epidemics of acute haemorrhagic conjunctivitis in the last decade by virus isolation from the conjuctiva or demonstration of antibody in the serum, the neurological and eye disease were linked only on strong clinical, epdidemiological and serological grounds, till we reported ${ }^{13}$ in our preliminary communication the presence of significant titres of antibody against EV 70 in the CSF of our neurologically afflicted patients. The final results of our observations briefly mentioned here and reported elsewhere ${ }^{14}$ suggest that there is de novo synthesis of the antibody against EV 70 in the CSF proving direct invasion of this virus or its antigenically similar local strain into the CNS. It has also been shown that the three types of polio viruses and the coxsackie virus A24 were not implicated in this epidemic.

It is known that other viruses especially coxsackie A24 and adenovirus can cause sporadic and epidemic conjunctivitis, and concurrent infection with EV 70 and coxsackie virus $A 24,{ }^{37}$ and picornavirus (reportedly similar to EV 70) and adenovirus type $2^{38}$ can occur in certain epidemics. It is also known that the acute paralytic illness caused by 21 types of enterovirus (polio, coxsackie, ECHO and ungrouped enterovirus EV 70, 71) ${ }^{39}$ cannot be identified as due to a specific virus on purely clinical grounds. Yet the combination of a haemorrhagic conjunctivitis especially in epidemic and pandemic proportions and an acute paralysis simulating classical poliomyelitis is caused by EV 70 alone.

The virus first grows in the epithelial cells of the conjuctiva and spreads mainly by the eye-handfomite route. Its spread from the conjunctiva to the CNS is not known, but it is believed to be similar to poliomyelitis, ${ }^{40}$ either via the blood stream or the neural route through nerves innervating the eyes. In this regard it is notable that six patients had indentical neurological disease without the conjunctivitis by mere close contact with patients with acute haemorrhagic conjunctivitis. It is likely that the conjunctival entry of the virus remained subclinical, or there was an alternate yet unrecognised portal of entry. Hung and $\mathrm{Kono}^{19}$ have similarly shown high antibody titres in the serum of children during an epidemic without any clinically obvious eye disease. The shorter latent interval between the eye disease and the cranial nerve paralysis, the occasional appearance of the neurological illness before the conjunctivitis, the unusually long latent interval in some patients, the appearance of the CNS disease without the preceding tell-tale conjunctivitis and the sparing of the nervous system in the vast majority (and more especially children) will be understood when one gains 
precise knowledge not only of the replication of the virus and its transmission to the nervous system, but also of host susceptibility to its various strains.

As experience so far has shown that epidemics will recur, it is conceivable that enhanced neurovirulence of the virus in a particular epidemic may cause proportionally more neurological cases, or paralytic cases without the conjunctivitis. A routine laboratory procedure should be established to estimate antibody titres against EV 70 in the serum and CSF of all patients, who have an acute anterior horn cell paralysis with or without conjuntivitis. This would help estimate the extent of the infection by this virus in both epidemic and sporadic forms, and provide a diagnostic test to rule out coincidental unrelated neurological illness following AHC.

We now feel that whereas the descriptive titles of "acute haemorrhagic conjunctivitis" and "lumbosacral radiculomyelitis" or "adult poliomyelitis" are useful to identify clinically the two parts of the disease, the combination should be called "Enterovirus 70 disease" because no other virus has so far been found to cause it. Similarly, if at the very beginning of an epidemic of conjunctivitis EV 70 is identified as the causative agent, then the subsequent cases should be designated also as "Enterovirus 70 disease". This would ensure more urgent health measures, and avoid accepting it as a benign self-limiting local eye disease rather than one which can cause a permanent disabling paralysis. A timely warning has already been sounded. ${ }^{41}$

We are grateful to Drs $\mathrm{R}$ Kono, $\mathrm{K}$ Miyamura, K Hashimoto, T Ogino, T Hikiji, of the National Institute of Health and Special Reference Laboratory, Tokyo, for carrying out the serological study and for permission to quote a part of the data. The technical assistance of Mr Deepak Sagar and Dr Meher Desai along with the staff of the Medical Education Department of Glaxo Laboratories (India) Limited is happily acknowledged.

\section{References}

1 Wadia NH, Wadia PN, Katrak SM, Misra VP. Neurological manifestations of acute haemorrhagic conjunctivitis. Lancet 1981;2:528-9.

${ }^{2}$ Wadia NH, Wadia PN, Katrak SM, Misra VP. Neurological manifestations of acute haemorrhagic conjunctivitis. In: Katsuki S, Tsubaki T, Toyokura Y, eds. Neurology, Amsterdam, Excerpta Medica 568, 1982;341-51.

${ }^{3}$ Centres for disease control. Acute haemorrhagic conjunctivitis. Florida, North Carolina. Morbid Mortal Wkly. Rep. 1981;30:501-2.

${ }^{4}$ Centres for disease control. Acute haemorrhagic con- junctivitis. Latin America. Morbid Mortal Wkly. Rep. 1981;30:450-1.

${ }^{5}$ Centres for disease control. Acute haemorrhagic conjunctivitis. Panama and Belize. Morbid Mortal Wkly. Rep. $1981 ; 30: 497-8$.

- Wadia NH, Irani PF, Katrak SM. Neurological complications of a new conjunctivitis. Lancet 1972;2:970-1.

${ }^{7}$ Wadia NH, Irani PF, Katrak SM. Lumbosacral radiculomyelitis associated with pandemic acute haemorrhagic conjunctivitis. Lancet 1973;1:350-2.

${ }^{8}$ Kono R, Sasagawa A, Ishii K, et al. Pandemic of new type of conjunctivitis. Lancet 1972;1:1191-4.

9 Kono R, Uchida N, Sasagawa A, et al. Neurovirulence of acute haemorrhagic conjunctivitis virus in monkeys. Lancet 1973;1:61-3.

${ }^{10}$ Kono R, Miyamura K, Tajiri E, et al. Neurological complications associated with acute haemorrhagic conjunctivitis virus infection and its serologic confirmation. J Infect Dis 1974;129:590-3.

"Shahani BT, Potts F, Domingue J. F response studies in peripheral neuropathy. Neurology (Minneap) 1980; 30:409-10.

12 Desmedt JE, Noel P. Average cerebral evoked potentials in the evaluation of lesions of the sensory nerves and of the central somatosensory pathways. In: Desmedt JE, ed. New Developments in Electromyography and Clinical Neurophysiology. Basel, Karger 1973;2:353-71.

${ }^{13}$ Kono R, Wadia NH, Miyamura K, et al. Antibody titres to enterovirus type 70 in the 1981 Indian epidemic of acute haemorrhagic conjunctivitis. Lancet 1981;2:924-5.

14 Wadia NH, Katrak SM, Misra VP, et al. Polio-like motor paralysis associated with acute haemorrhagic conjunctivitis in the 1981 outbreak of Bombay: Clinical and Serological studies. J Infect Dis 1983 (in press).

${ }^{15}$ Bharucha EP, Mondkar VP. Neurological complications of a new conjunctivitis. Lancet 1972;2:970-1.

${ }^{16}$ Bujarborua D, Dutta LC, Dutta NN. Epidemic conjunctivitis. Orient Arch Opthal 1972;10:58-63.

${ }^{17}$ Saxena RC, Bhatia A, Chaturvedi UC. Recent epidemic conjunctivitis in Lucknow: A clinical study Orient Arch Ophthal 1972;10:253-7.

${ }^{18}$ Hung TP, Sung SM, Liang HC, Landsborough D, Green IJ. Radiculomyelitis following acute haemorrhagic conjunctivitis. Brain 1976;99:771-90.

${ }^{19}$ Hung TP, Kono R. Neurological complications of acute haemorrhagic conjunctivitis (a polio-like syndrome in adults). In: Vinken PJ, Bruyn GW, eds. Handbook of Clinical Neurology, Vol. 38, Amsterdam: North Holland, 1979;595-623.

${ }^{20}$ Kono R, Miyamura K, Tajiri E, Robin Y, Girard P. Serological studies of radiculomyelitis occurring during the outbreak of acute haemorrhagic conjunctivitis in Senegal in 1970. Jap J Med Sci Biol 1976;29:91-4.

${ }^{21}$ Phuapradit $P$, Roongawithu U, Limsukon P, Boongird P, Vejjajiva A. Radiculomyelitis complicating acute haemorrhagic conjunctivitis. A clinical study.J Neurol Sci 1976;27:117-22.

${ }^{22}$ Kuroiwa Y, Tsuji S, Murai Y, Shibasảki H. A case of poliomyelitis syndrome following acute haemorrhagic conjunctivitis, the first Japanese case. Neurol Med 
(Tokyo) 1977;7:87-8.

${ }^{23}$ Kono R, Miyamura K, Tajiri E, et al. Virological and serological studies of neurological complications of acute haemorrhagic conjunctivitis in Thailand. $J$ Infect Dis 1977;135:706-13.

${ }^{24}$ Kono R, Sasagawa A, Miyamura K, Tajiri E. Serologic characterization and sero-epidemiologic studies on acute haemorrhagic conjunctivitis (AHC) virus. Am J Epidemiol 1975;101:444-57.

${ }^{25}$ Quarcoopome CO, Hosaka A, Uchida Y, Sasagawa A, Kono R. Clinico-epidemiologic studies of acute haemorrhagic conjunctivitis in Ghana. Jap $J$ Ophthalmol 1979;23:119-25.

${ }^{26}$ Thakur LC. Cranial nerve paralysis associated with acute haemorrhagic conjunctivitis. Lancet 1981;2:584.

${ }^{27}$ Mondkar VP. Cranial nerve paralysis associated with acute haemorrhagic conjunctivitis. Lancet $1981 ; 2: 584$.

${ }^{28}$ Katiyar BC, Misra S, Singh RB, Singh AK. Neurological Syndrome after acute epidemic conjunctivitis. Lancet 1981;2:866-7.

${ }^{29}$ Hodes R. As quoted by PM Le Quesne. Nerve conduction study. In: Licht S. ed. Electrodiagnosis ana Electromyography. Baltimore, Maryland, Waverley Press Inc. 1971;2:419-51.

${ }^{30}$ Lambert EH. As quoted by PM Le Quesne. Nerve conduction study. In: Licht S. ed. Electrodiagnosis and Electromyography. Baltimore, Maryland, Waverley Press Inc. 1971;2:419-51.

${ }^{31}$ Shahani BT, Young RR. Studies of reflex activity from a clinical view point. In: Aminoff MJ. ed. Electrodiagnosis in Clinical Neurology. New York, Churchill Livingstone 1980;290-301.

${ }^{32}$ Albizzati MG, Bassi S, Passerini D, Crespi V. F wave velocity in motor neuron disease. Acta Neurol Scand 1976;54:269-77.

${ }^{33}$ Schiller HH. Nature and pathophysiology of the F wave, a single fibre EMG investigation. Electroencephal and Clin Neurophysiol 1980;48:17.

${ }^{34}$ Price RW, Plum F. Poliomyelitis. In: Vinken PJ, Bruyn GW. eds. Handbook of clinical neurology. Vol. 34, Amsterdam, North Holland 1978, 93-132.

35 Wadia RS, Divate PG, Sowani AP, Das. Neurological Syndrome after epidemic conjunctivitis. Abstracts XXXI Annual Conference of the Neurological Society of India, Visakhapatnam 1981;53.

${ }^{36}$ Kar AM, Nag D, Mehrotra A, Shukla R, Misra UK. Neurological sequelae of epidemic conjunctivitis in Uttar Pradesh. Abstracts XXXI Annual Conference of the Neurological Society of India, Visakhapatnam $1981 ; 52$.

${ }^{37}$ Lim KH, Yin-Murphy M. The aetiological agents of epidemic conjunctivitis. Singapore Med J 1977;18:41-3.

${ }^{38}$ Chaturvedi UC, Mathur, A, Singh UK, Kapoor AK, Mehrotra RML, Saxena RC. An epidemic of picornavirus and adenovirus conjunctivitis. $\mathrm{Br} J$ Ophthalmol 1975;59:439-43.

${ }^{39}$ Sabin AB. Paralytic poliomyelitis: Old dogmas and new perspectives. Rev Infect Dis 1981;3:543-64.

${ }^{40}$ Kono R, Wadia NH, Hung TP, Vejjajiva A. Neurologic complications of acute haemorrhagic conjunctivitis: Virologic aspects. In: Katsuki S, Tsubaki T, Toyokura Y. eds. Neurology, Amsterdam, Excerpta Medica 568, 1982;352-8.

${ }^{41}$ Editorial. Neurovirulence of Enterovirus 70. Lancet 1982;1:373-4. 